Regisseur Olivier Py mit drastischen Bildern auf die Bühne, so, als wollte er die zur Zeit in Frankreich heiß umstrittene Debatte um die Homosexuellen-Ehe anheizen. In der zweiten Szene wird Albin das Opfer einer brutalen Massenvergewaltigung. Es gibt eine kurze und heftige Liebesszene zwischen Albin und Claude. Am Rande wird diese Oper also tagesaktuell. Denn nach der Premiere steht ein Polizeigebot vor der Oper und hält eine Demonstration von den herausströmenden Besuchern fern. Unter ihnen die derzeitige Justizministerin, Christiane Taubira. Und das auch am Rande: Homosexualität ist für Robert Badinter als Justizminister ebenfalls ein Thema gewesen. Denn er hat als Justizminister die Entkriminalisierung der Homosexualität in der Gesellschaft durchgesetzt. In dem Musikdrama "Claude" geht es aber vorrangig um die Konfrontation zwischen einem gnadenlosen Vorgesetzten und seinem ihm hilflos ausgelieferten Untergebenen. „Ein Justizvollzug muss den Menschen führen, formen, bilden und auf die Reintegration hinarbeiten. “ Da hat Robert Badinter ganz klare Vorstellungen. Und Victor Hugo hat das schon im 19. Jahrhundert gefordert. Eine Gesellschaft, die Verlierer produziert, gefährdet sich selbst. Die Wirtschaftskrise und Arbeitslosigkeit, die zurzeit in Frankreich auf einen Rekord zusteuert, sind eine große Gefahr. Wie gut, dass es Menschen wie Robert Badinter gibt, die nicht aufhören, ihre Stimme zu erheben. Solche Leute braucht die Gesellschaft, braucht die Justiz! Und Opernfestivals, die so mutig sind wie in Lyon, solche Themen auf die Bühne zu bringen.

\title{
Rechtsprechung
}

\section{Keine Rückgabe von Antiken an die Türkei}

OLG Frankfurt am Main, Urteil vom 04. Februar 2013 - 16 U 161/11

\begin{abstract}
Aus der Tatsache, dass der Veräußerer keine Papiere (Fundanzeige, Grabungsgenehmigung) beifügt, ist nicht zwingend zu schließen, dass er kein Eigentümer ist, wenn der Fundort unbekannt ist und der Wert der Gegenstände gering erscheint. (Leitsatz der Redaktion)
\end{abstract}

\section{Gründe}

\section{I.}

- Die Parteien streiten um die Herausgabe von antiken Schalen und Gefäßen sowie um einen Unterlassungsanspruch der Klägerin bzgl. des Handels mit dem türkischen Kulturschutzgutrecht unterliegenden Objekten.

Diese Objekte sind von der hessischen Polizei im Jahr 2008 beim Beklagten beschlagnahmt und mit Verfügung des Hessischen Ministeriums für Wissenschaft und Kunst vom 16. Dezember 2009 sichergestellt worden. Im Rahmen eines vom Amtsgericht Frankfurt gegen den Beklagten geführten Ermittlungsverfahrens wegen Hehlerei gab der Beklagte an, die Gegenstände im Jahr 2007 in X1 von einem Ehepaar A erworben zu haben. Die Eheleute A haben diesen Vortrag in ihrer polizeilichen Vernehmung vom 01. Oktober 2008 vor dem ... Landeskriminalamt bestätigt und angegeben, von 1975 bis 1980 in X2 gelebt zu haben, wo der Zeuge als Lehrer an der deutschen Schule tätig war. Die streitgegenständlichen Objekte sollen nach den polizeilichen Angaben der Eheleute A ihnen im Jahr 1980 von einem armenischen Teppichhändler gemeinsam mit zwei Teppichen als Geschenk nach Deutschland nachgeschickt worden sein. Das Landgericht Frankfurt hat mit Beschluss vom 04. Dezember 2009 die Beschlagnahme der Gegenstände aufgehoben und festgestellt, dass wegen feststehenden Erwerbs des Beklagten von den Eheleuten A kein Tatverdacht bestehe. Mit
Urteil des Verwaltungsgerichts Frankfurt am Main vom 02. Juni 2010 (5 K 1082/10.F) wurde das Land Hessen verurteilt, die sichergestellten Gegenstände an den Beklagten herauszugeben.

Die Klägerin ist der Ansicht gewesen, sie sei auf der Grundlage des geltenden türkischen Kulturgüterschutzgesetzes als Eigentümerin der streitgegenständlichen Objekte anzusehen. Sie hat behauptet, die Objekte würden aus der Türkei stammen.

Das Landgericht hat durch Urteil vom 18. August 2011 die Klage abgewiesen.

Zur Begründung hat es ausgeführt, der Beklagte könne die Eigentumsvermutung des Besitzers nach $\S 1006$ Abs. 1 BGB für sich in Anspruch nehmen. Diese seien nicht von der Klägerin widerlegt, weil ein Abhandenkommen im Sinne von § 1006 Abs. 1 S. 2 BGB nicht bewiesen sei. Dazu sei ein Besitz der Klägerin notwendig, der aber niemals bestanden habe. Auch habe die Klägerin den Erwerb des Beklagten von den Eheleuten A im Jahr 2007 nicht substantiiert bestritten. Jedenfalls habe der Beklagte gutgläubig Eigentum erworben.

Gegen dieses der Klägerin am 24. August 2011 zugestellte Urteil hat sie mit einem am 16. September 2011 eingegangenen Schriftsatz Berufung eingelegt, die sie mit einem am 21. Oktober 2011 eingegangenen Schriftsatz begründet hat. 
Zur Begründung ihres Rechtsmittels führt die Klägerin aus, sie sei Eigentümerin und habe dieses Eigentum durch eine sachverständige Stellungnahme belegt. Das Landgericht hätte die Eheleute A persönlich vernehmen müssen. Der Beklagte habe auch nicht gutgläubig erworben, da er als Antiquitätenhändler die Anforderungen an Genehmigungspapiere kenne. Auch seien die Gegenstände der Klägerin abhanden gekommen.

Die Klägerin beantragt,

1. unter Aufhebung des Urteils des Landgerichts Frankfurt Az. 2-13 O 212/10 vom 30. Juni 2011 den Beklagten zu verurteilen, die in seinem Besitz befindlichen drei vorchristlichen phrygischen Omphalosschalen und zwei byzantinischen Hängegefäße an die Klägerin herauszugeben,

2. unter Aufhebung des Urteils des Landgerichts Frankfurt 2-13 O 212/10 vom 30. Juni 2011 den Beklagten zu verurteilen, es bei Meidung eines Ordnungsgelds bis zu 250.000 Euro wahlweise bis zu 6 Monate Ordnungshaft zu unterlassen, mit beweglichen, schutzwürdigen türkischen Kulturgütern im Sinne des $\S 23$ des türkischen Gesetzes zum Schutz von Kultur- und Naturgut vom 21. Juli 1983 in der Fassung vom 14. Juli 2004 Handel zu treiben, wenn hierfür im Einzelfall keine schriftliche Bewilligung des türkischen Kultur- und Tourismusministeriums gemäß § 26 dieses Gesetztes vorliegt.

Der Beklagte beantragt,

die Berufung zurückzuweisen.

Er ist der Ansicht, die Klägerin sei nie Eigentümerin der Schalen gewesen. Die Schalen seien auch nie auf dem Gebiet der Türkei gefunden worden. Bei den Schalen handele es sich um antike Massenware, die im gesamten östlichen Mittelmeer gefunden wurde. Die Klägerin habe die Fristen des Kulturgüterrückgabegesetzes nicht beachtet. Er könne nicht zur Einhaltung türkischen Rechts in Deutschland verpflichtet werden.

\section{II.}

Die Berufung der Klägerin ist zulässig. Sie ist insbesondere form- und fristgerecht eingelegt und begründet worden.

Das Rechtsmittel der Klägerin ist aber unbegründet. Zu Recht hat das Landgericht die Klage abgewiesen. Der Klägerin steht gegen den Beklagten kein Anspruch auf Herausgabe der streitgegenständlichen Schalen und Gefäße zu.

Ein solcher Anspruch ergibt sich nicht aus $\S 6$ Abs. 2 KultGüRückG, da dieser Anspruch gemäß $§ 13$ Abs. 1 KultGüRückG vor dem Verwaltungsgericht geltend zu machen ist. Außerdem gilt dort die Ein-Jahresfrist zur Erklärung der Gegenstände als für Archäologie besonders bedeutsam, beginnend mit dem Zeitpunkt, an dem die Klägerin Kenntnis von den Gegenständen erlangen konnte. Dass die Klägerin diese Frist eingehalten hat, ist nicht erkennbar. Es fehlt sogar an der Behauptung der Klägerin, die Gegenstände in ein besonderes Verzeichnis der besonders schutzwürdigen Kulturgüter aufgenommen zu haben.

Die Klägerin hat gegen den Beklagten auch keinen Anspruch aus § 985 BGB. Diese Vorschrift setzt voraus, dass die Klägerin Eigentümerin und der Beklagte unrechtmäßiger Besitzer der Schalen und Gefäße ist.

Der Beklagte hat zwar behauptet, nicht mehr im Besitz der Gegenstände zu sein. Hierauf kommt es aber nicht, da die Klägerin ihr Eigentum nicht bewiesen hat.

Es erscheint bereits zweifelhaft, ob die Klägerin zu irgendeinem Zeitpunkt einmal Eigentümerin war. Als Erwerbstatbestand kommen $\S 3$ des Antikengesetzes und § 5 des Gesetzes zum Schutz von Kultur- und Naturgut in Betracht. Diese türkischen Vorschriften setzen nicht nur voraus, dass es sich bei den Schalen um Kulturgüter handelt, sondern dass sie auf dem Boden der Türkei gefunden bzw. ausgegraben wurden und dass dies nicht vor Inkrafttreten der beiden Gesetze geschehen ist. Nach dem ersten türkischen Kulturschutzgesetz von 1869 war Eigentümer des Kulturgutes derjenige, auf dessen Grundstück das Kulturgut gefunden wurde. Erst durch das Kulturgüterschutzgesetz von 1874 wurde Staatseigentum von unentdeckten Kulturgütern begründet. In dem weiteren Kulturgüterschutzgesetz von 1884 wurde auch die Möglichkeit von Privateigentum bei zufälligen Funden geregelt. Erst durch das Gesetz von 1906 wurde die vorgenannte Ausnahme abgeschafft. Alle Kulturgüter, welche sich auf privaten oder öffentlichen Grund befinden, sind per Gesetz Eigentum des türkischen Staates. Allerdings hatte das Gesetz keine Rückwirkung, so dass vorhergehende Funde nicht zwingend zu Alleineigentum des Staates führten.

Auch das Antikengesetz von 1973 sah das Eigentum des Staates an allen beweglichen Kulturgütern vor, ermöglichte aber eine Rückgabe an den Grundstückseigentümer, falls die Gegenstände nicht registriert wurden. Diese Rückgabe stellte einen Verzicht auf das Eigentum dar. Gleiches gilt für das Gesetz zum Schutz von Kultur- und Naturgut vom 21. Juli 1983.

Der Beklagte hat insoweit bestritten, dass die Gegenstände überhaupt jemals auf türkischem Boden ausgegraben wurden, da derartige Schalen im gesamten östlichen Mittelmeer gefunden wurden. Aus dem Vorbringen der Klägerin ist nicht sicher zu entnehmen, wann die Schalen überhaupt gefunden wurden, ob dies auf privatem oder öffentlichem Grund geschehen ist und welches Recht insoweit anwendbar ist. Ohne detaillierte Darlegung mit entsprechenden Beweisangeboten, wann diese Gegenstände wo ausgegraben wurden, ist eine rechtliche Beurteilung dazu, ob die Klägerin jemals Eigentümerin der Schalen und Gefäße war, nicht möglich. 
Aber selbst wenn man unterstellen würde, dass die Klägerin irgendwann nach irgendwelchen türkischen Vorschriften Eigentum an den Gegenständen erworben hätte, hätte sie dieses verloren durch gutgläubigen Erwerb der Eheleute $A$.

Zu Recht geht das Landgericht in dem angefochtenen Urteil davon aus, dass sich die Frage des Eigentumserwerbs nach deutschem Recht richtet. Nach § 43 Abs. 1 EGBGB entscheidet über Erwerb, Inhalt und Verlust des Eigentums die Rechtsordnung des Staates, in dem sich die Sache befindet. Dies gilt auch für Kulturgüter (Palandt-Thorn, BGB, 71. Aufl. 2012 Art. 43 EGBGB). Darüber besteht in zweiter Instanz zwischen den Parteien auch kein Streit. Nach dem Vorbringen des Beklagten, das durch die Aussage der Zeugin A2 bestätigt wurde, haben die Eheleute A im Jahre 1980 die Schalen und Gefäße von einem armenischen Teppichhändler als Zugabe wegen der verspäteten Lieferung von gekauften Teppichen nach Deutschland erhalten. Mit der Übersendung und Übergabe sowie der Entgegennahme der Gegenstände durch die Eheleute A in Deutschland ist eine Einigung über den Eigentumsübergang erfolgt sowie eine Übergabe. Diese Einigung über den Eigentumsübergang verstößt nicht gegen § 134 BGB und damit nicht gegen ein gesetzliches Verbot, da ausländische Gesetze keine Verbotsgesetze im Sinne dieser Vorschrift sind (BGHZ 59, 85).

Aber auch eine Nichtigkeit der dinglichen Einigung nach $\S 138$ Abs. 1 BGB liegt nicht vor. Das Anstandsgefühl aller billig und gerecht Denkenden ist nicht verletzt, wenn ein Händler aus einem türkischen Basar als Zugabe zum Teppichkauf fünf antike Schalen übereignet. Das dingliche Geschäft ist wertneutral. Die Nichtigkeit des Kausalgeschäfts schlägt in der Regel nicht durch auf das dingliche Geschäft (BGH NJW 1990, 384 [BGH 03. Oktober 1989 - XI ZR 154/88]). Anders ist dies nur, wenn die Unsittlichkeit gerade im Vollzug der Leistung liegt (BGH NJW-RR 2006, 888 [BGH 20. Januar 2006 - V ZR 214/04]). Dies ist indes nicht der Fall. Das ergibt sich bereits aus $\S 6$ des KultGüRückG. Danach kann die Rückgabe unrechtmäßig erlangter Kulturgüter anderer Staaten nur unter sehr engen Voraussetzungen innerhalb knapper Fristen verlangt werden. Dieser Umstand zeigt ebenso wie die Tatsache, dass ausländische Gesetze keine Nichtigkeit deutscher Rechtsgeschäfte herbeiführen können, dass es keinesfalls sittenwidrig sein kann, fremde Kulturgüter zu übereignen.

Es ist auch grundsätzlich davon auszugehen, dass die Eheleute A das Eigentum von dem armenischen Händler als Berechtigtem erworben haben. Da er ursprünglich Besitzer der Gegenstände war, wird gem. § 1006 Abs. 1 BGB vermutet, dass er auch Eigentümer der Gefäße war. Ausdrücklich hat die Klägerin nicht behauptet, dass der armenische Händler kein Eigentümer war. Selbst wenn man in ihrem Bestreiten der Ereignisse aus dem Jahre 1980 hilfsweise die Behauptung entnehmen wollte, der armenische Händler sei entgegen der gesetzlichen Vermutung nicht Eigentümer gewesen, genügt dieses Vorbringen nicht, da die Klägerin nach allgemeiner Ansicht (vgl. Palandt-Bassenge,
72. Aufl., § 932 Rz. 15 mwN) darlegungs- und beweisbelastet ist für das Nichteigentum des Veräußerers. Einen Beweis hat sie insoweit nicht einmal angeboten.

Selbst wenn man das Beweisangebot bzgl. der Bösgläubigkeit der Eheleute A auch als Beweisangebot bzgl. des fehlenden Eigentums des armenischen Teppichhändlers auffassen würde, wäre der Beweis nicht erbracht. Aus der Aussage der Zeugen A2 sind keine Angaben zu entnehmen, die darauf schließen lassen, dass der armenische Händler kein Eigentümer der übersandten Gegenstände war. Aber selbst wenn man weiterhin unterstellen würde, dass der armenische Händler nicht Eigentümer der Schalen war, hätte die Klägerin etwaiges Eigentum verloren durch den gutgläubigen Erwerb der Eheleute A. Die Klägerin hat zwar behauptet, die Eheleute A seien hinsichtlich des Eigentums des armenischen Teppichhändlers bösgläubig gewesen. Darlegungs- und beweisbelastet für die Bösgläubigkeit der Eheleute $A$ ist aber die Klägerin (BGH NJW 1982, 38 [BGH 05. Oktober 1981 - VIII ZR 235/80]). Diesen Beweis hat die Klägerin nicht erbracht. Zum Beweis für die Bösgläubigkeit hat sich die Klägerin auf das Zeugnis der Eheleute A berufen. Diese haben aber gerade nicht bestätigt, dass sie wussten, dass der armenische Händler nicht Eigentümer der Gefäße und Schalen war. Auch eine grobe Fahrlässigkeit der Eheleute $A$ ist nicht nachgewiesen. Eine grobe Fahrlässigkeit setzt voraus, dass die im Verkehr erforderliche Sorgfalt in besonders schwerem Maße verletzt ist und dass dasjenige nicht beachtet wurde, was unter den gegebenen Umständen jedem hätte einleuchten müssen. Die Zeugin A2 hat bei ihrer Vernehmung ausdrücklich ausgesagt, sie hätte sich wohl keine Gedanken darüber gemacht, ob es illegal gewesen sein könnte, dass die Gegenstände aus der Türkei nach Deutschland verbracht worden sind. Sie hätten kein Unrechtsgefühl gehabt. Mit dieser auf Antrag der Klägerin vernommenen Zeugin konnte der Beweis der Bösgläubigkeit nicht erbracht werden. Die Klägerin hat die Angaben der von ihr benannten Zeugin selbst als unglaubwürdig angesehen. Dadurch ist allerdings nicht das Gegenteil dessen bewiesen, was die Zeugin ausgesagt hat. Auch aus den äußeren Umständen des Erwerbs ist nicht zu schließen, dass die Zeugin A bösgläubig war hinsichtlich des Eigentums des armenischen Händlers. Aus der Tatsache, dass der armenische Händler seinem Geschenk keine Papiere (Fundanzeige, Grabungsgenehmigung oder ähnliches) beigefügt hat, lässt sich nicht zwingend schließen, dass der Veräußerer kein Eigentümer ist. Das gilt insbesondere wenn man berücksichtigt, dass die Eheleute A nicht sicher wussten, ob die Gegenstände tatsächlich auf türkischem Boden gefunden wurden, der Wert der Waren nicht außergewöhnlich hoch war, da sie als Zugabe übersandt wurden und später von innen für nur 200 Euro weiter verkauft worden sind. Insoweit haben die Eheleute $A$ sich nicht grob fahrlässig verhalten, als sie die in ihren Augen geringwertigen Waren ohne Nachforschungen nach Papieren entgegennahmen.

Einen gutgläubigen Erwerb der Eheleute $A$ steht auch nicht entgegen, dass die streitgegenständlichen Schalen und Gefäße der Klägerin gestohlen oder sonst abhanden gekommen sind. 
Ein Abhandenkommen im Sinne von § 935 Abs. 1 BGB liegt nur vor, wenn der Eigentümer den Besitz an einer Sache ohne seinen Willen verliert (Palandt-Bassenge, § 935 Rz. 3 mwN). Die Klägerin hatte aber niemals unmittelbaren Besitz an den Gegenständen.

Im Übrigen hätten die Eheleute A das Eigentum auch nach § 937 BGB erworben, da sie die Gegenstände über 10 Jahre im Eigenbesitz hatten und nicht bösgläubig waren, wie bereits dargelegt wurde.
Es konnte deshalb auch offen bleiben, ob der Beklagte überhaupt noch im Besitz der Gefäße und Schalen ist. Insoweit hatte er schon in erster Instanz vorgetragen, die Gegenstände weiter veräußert zu haben.

Zutreffend hat das Landgericht auch den Klageantrag zu 2) abgewiesen. Auf die diesbezüglichen Ausführungen in den Entscheidungsgründen des angefochtenen Urteils wird Bezug genommen. (Entscheidung von der Redaktion bearbeitet.)
Da die Eheleute A Eigentum erworben und der Beklagte die Gegenstände käuflich erworben hat, hat er das Eigentum von den Berechtigten erworben, so dass es auf seine Gut- oder Bösgläubigkeit nicht ankommt.

\section{Rückgabe von Raubkunst an die Republik Zypern}

OLG München, Urteil vom 18. März 2013 - 19 U 4878/10

1. Die Republik Zypern hat ihr Eigentum an den streitgegenständlichen Kunstgegenständen nicht durch die Besetzung Nordzyperns durch die Türkische Republik Nordzypern verloren. Diese kann keine Rechtspersönlichkeit sein, weil sie von der Staatengemeinschaft nicht als völkerrechtliches Subjekt anerkannt wird.

2. Ansprüche auf Herausgabe beschlagnahmter Gegenstände sind nach Abschluss des Strafverfahrens auf dem Zivilrechtsweg geltend zu machen. (Leitsätze der Redaktion)

\section{Gründe:}

\section{I.}

- Die Klägerinnen begehren die Zustimmung des Beklagten zur Herausgabe von Ikonen, Kirchenkunst und frühgeschichtlichen Gegenständen, die die Staatsanwaltschaft München I im Rahmen des gegen den Beklagten wegen Hehlerei geführten Ermittlungsverfahrens 237 Js 230262/97 sichergestellt hat und die derzeit im Bayerischen Landeskriminalamt ... gelagert sind.

Die Klägerinnen berufen sich dabei auf ihr Eigentumsrecht an den genannten Gegenständen.

Sie tragen vor, dass sich die kirchlichen Gegenstände bis zur Besetzung des nördlichen Teil Zyperns durch türkische Truppen im Jahre 1974 dort befunden hätten. Sie seien in der Zeit danach aus den Kirchen, Klöstern und Museen im nördlichen Teil Zyperns entfernt und außer Landes gebracht worden. Die ebenfalls sichergestellten, aus Ausgrabungen stammenden prähistorischen Gegenstände, stünden aufgrund des geltenden Antiquities Law im Eigentum der Republik Zypern.

Der Beklagte ist der Auffassung, er bzw. seine Tochter oder seine Ehefrau seien Eigentümer der sichergestellten Gegenstände.
Auf Antrag der Staatsanwaltschaft München I hat der zuständige Strafsenat des Oberlandesgerichts München bereits am 3. Juni 2004 entschieden, dass einige der Gegenstände im Wege der Rechtshilfe an die Klägerinnen herauszugeben seien. Das Auswärtige Amt der Bundesrepublik Deutschland genehmigte die Rechtshilfe allerdings nicht und verwies die Klägerinnen auf den Zivilrechtsweg.

Aufgrund der daraufhin erhobenen Zivilklage hat das Landgericht München I mit der angefochtenen Entscheidung nach Durchführung der Beweisaufnahme der Klage in vollem Umfange stattgegeben.

In seiner Entscheidung stützt sich das Erstgericht zunächst darauf, dass die Kläger Eigentümer der asservierten Gegenstände seien und dem Beklagten kein Recht auf Herausgabe an ihnen zustehe. Es folgt aufgrund der durchgeführten Beweisaufnahme den Ausführungen des Sachverständigen Prof. D., wonach sich bestimmte Gegenstände bis 1974 in zypriotischen Kirchen, Klöstern und Kunstmuseen befunden hätten.

Auch soweit eine Zuordnung durch den Sachverständigen nicht eindeutig möglich gewesen sei, könnten die Klägerinnen die Zustimmung zur Herausgabe verlangen. Die Staatsanwaltschaft sei bereits dann zur Herausgabe an die Klägerinnen berechtigt, wenn feststünde, dass dem Beklagten keine Rechte an 